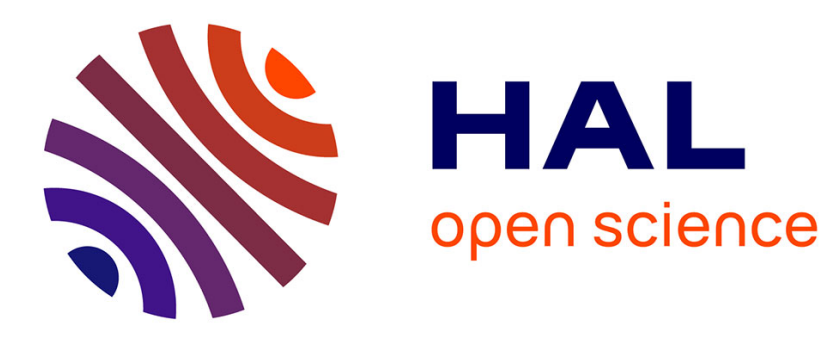

\title{
Sriwijaya, entre texte historique et terrain archéologique: un siècle à la recherche d'un État évanescent
}

Pierre-Yves Manguin

\section{- To cite this version:}

Pierre-Yves Manguin. Sriwijaya, entre texte historique et terrain archéologique: un siècle à la recherche d'un État évanescent. Bulletin de l'Ecole française d'Extrême-Orient, 2001, 88 (1), pp.331339. 10.3406/befeo.2001.3522 . halshs-02509103

\section{HAL Id: halshs-02509103 \\ https://shs.hal.science/halshs-02509103}

Submitted on 16 Mar 2020

HAL is a multi-disciplinary open access archive for the deposit and dissemination of scientific research documents, whether they are published or not. The documents may come from teaching and research institutions in France or abroad, or from public or private research centers.
L'archive ouverte pluridisciplinaire HAL, est destinée au dépôt et à la diffusion de documents scientifiques de niveau recherche, publiés ou non, émanant des établissements d'enseignement et de recherche français ou étrangers, des laboratoires publics ou privés. 
Sriwijaya, entre texte historique et terrain archéologique : un siècle à la recherche d'un État évanescent

Pierre-Yves Manguin

\section{Citer ce document / Cite this document :}

Manguin Pierre-Yves. Sriwijaya, entre texte historique et terrain archéologique : un siècle à la recherche d'un État évanescent. In: Bulletin de l'Ecole française d'Extrême-Orient. Tome 88, 2001. pp. 331-339;

doi : https://doi.org/10.3406/befeo.2001.3522

https://www.persee.fr/doc/befeo_0336-1519_2001_num_88_1_3522

Fichier pdf généré le 08/11/2019 


\title{
Sriwijaya, entre texte historique et terrain archéologique : un siècle à la recherche d'un État évanescent
}

\author{
Pierre-Yves MANGUIN \\ «Personne ne sait mieux que toi, sage Kublaï, qu’il ne faut jamais \\ confondre la ville avec le discours qui la décrit " \\ Italo Calvino, Les villes invisibles, Paris, Le Seuil, 1974. \\ "The terrain is now the superior text in Sriwijayan studies" \\ Oliver W. Wolters, « Restudying Some Chinese Writings on Sriwijaya », 1986.
}

La lecture un tant soit peu attentive des travaux que l'historien Oliver Wolters a consacrés à l'étude de Sriwijaya - ce premier grand État maritime du monde malais, entre le $\mathrm{VII}^{\mathfrak{c}}$ et le XIII' siècles - révèle nettement qu'une rupture s'est produite dans son œuvre à la toute fin des années soixante-dix ${ }^{1}$. L'auteur de l'étude capitale sur les origines de Sriwijaya (Early Indonesian Commerce, Cornell University Press, 1967), étude fondée pour l'essentiel sur l'analyse des sources chinoises, éprouve alors le besoin de revenir sur ses pas. Le premier d'entre les quatre articles qu'il consacre presque coup sur coup entre 1979 et 1986 à l'étude de l'État malais et de sa localisation à Sumatra-Sud affirme sans ambages : "Je constate aujourd'hui que mon travail doit être révisé »" Les titres des trois autres articles réaffirment sans ambiguïté ce nécessaire retour en arrière : on y trouve en effet les termes «re-considération», "re-visiter» et "re-étudier » ${ }^{3}$. La raison du revirement qui nous vaut cet ensemble cohérent de travaux de révision, entrepris avant de se tourner désormais vers les études vietnamiennes, est révélé à sa lecture : poussé par des archéologues indonésiens - en particulier par $\mathrm{M}^{\text {me }}$ Satyawati Suleiman - et par quelquesuns de ses étudiants historiens et archéologues de Cornell University, Oliver Wolters s'est pour la première fois rendu en 1978 à Sumatra-Sud, berceau présumé de Sriwijaya ${ }^{4}$; il y retournera à plusieurs reprises jusqu'en 1986.

La révélation du "terrain » sumatranais, après quelque deux décennies de travail en bibliothèque, l'a en effet amené à revisiter les textes chinois et malais dont il était déjà familier, et à réviser nombre de ses conclusions antérieures sur la structure et l'environnement des sites anciens de la ville portuaire de Palembang, où il situe, comme bien d'autres avant lui, la place centrale de l'ancien État maritime.

1. Le professeur Oliver W. Wolters est décédé aux États-Unis le 5 décembre 2000, quelques heures après la lecture de la communication dont ce texte est une version légèrement remaniée. Sans le savoir, je rendais ainsi un dernier hommage à celui qui a le plus contribué à former la vision de l'histoire de l'Asie du Sud-Est qui est aujourd'hui la mienne - à la seule lecture de ses travaux d'abord, puis, après 1979, lors d'échanges personnels fréquents sur le terrain, à Cornell University ou épistolaires. En cela, je ne suis pas le seul : Oliver Wolters, par l'excellence de ses travaux, par ses qualités pédagogiques et, surtout, par sa très grande honnêteté intellectuelle, a formé ou influencé plusieurs générations d'historiens de l'Asie du Sud-Est, toutes nationalités confondues.

2. Wolters 1979 a, p. 3 : « Today I realize that my work needs revision ».

3. Wolters $1979 \mathrm{~b}, 1984,1986$.

4. Wolters, $1979 \mathrm{~b}$; on consultera aussi le récit de ce voyage à Sumatra, écrit par ses compagnons de terrain, dans McKinnon \& Milner, 1979. 
Cet épisode, pour anecdotique qu'il paraisse, est à bien des égards (re)fondateur de l'historiographie de Sriwijaya. Car un savant spécialiste du texte écrit y conclut à l'impossibilité pour lui de progresser plus avant sans laisser parler un autre texte, non écrit celuilà : le terrain. Pour Oliver Wolters, c'est désormais aux spécialistes de l'archéologie historique d'interpréter le discours que ce terrain fait entendre.

Avec la citation sibylline donnée en exergue, qui est tirée de ce dernier article de 1986 qu'Oliver Wolters consacre à l'étude de Sriwijaya, il passe en quelque sorte la main aux archéologues qui ont repris leurs recherches sur la région.

Il y a eu aussi chez lui, indéniablement, une part d'émerveillement à la découverte à partir de 1978 du terrain concret, physique, de Sumatra-Sud, découverte grâce à laquelle il perçoit dans les sources écrites de nouvelles résonances, plus riches, plus précises qu'il ne l'avait pressenti depuis son bureau ${ }^{5}$. Sa relecture minutieuse, à la lumière de ce nouvel éclairage, des notes prises par le fameux pèlerin chinois Yijing lors de ses séjours à Sriwijaya dans les années 670-690, en fournit un parfait exemple ${ }^{6}$.

Le constat de cette complémentarité première du «texte » et du « terrain » n'est pas en soi d'une grande nouveauté, même si elle n'a pas jusqu'alors été mise en pratique dans l'étude de l'histoire ancienne de Sumatra (si ce n'est par des non-spécialistes, tel le Résident Westenenk dans les années vingt, sans qu'ils ne soient pris bien au sérieux) ${ }^{7}$. C'est même cette complémentarité qui a, de fait, présidé à la fondation de l'EFEO au début de ce siècle : de façon explicite, on s'est alors attaché à combler le fossé creusé au $\mathrm{XIX}^{\mathrm{e}}$ siècle entre recherche en bibliothèque et connaissance de terrain, en créant de toutes pièces une institution qui allait faire vivre ses membres sur le terrain indochinois, au cœur de l'Asie orientale.

Néanmoins, la mise en œuvre de cette complémentarité élémentaire entre texte et terrain ne résout pas tous les problèmes de lecture des sources écrites. En restant dans le domaine de l'archéologie historique, il suffirait pour s'en convaincre de citer la multitude de rapports sur des fouilles effectuées en Indochine jusqu'aux années quarante, dont la fonction première était de fournir, littéralement, des illustrations pour accompagner le discours austère des philologues.

Avant de passer le flambeau de l'étude de Sriwijaya aux archéologues, Oliver Wolters, homme de textes, requiert la relecture de ces derniers : pour que les sources écrites puissent être mises en regard du système de signifiants constitué par le terrain sumatranais, il faut qu'elles soient elles-mêmes relues à l'aune des critères qui avaient présidé à leur rédaction, en mettant en branle toutes les techniques de la critique littéraire moderne : leurs caractéristiques narratives, les conventions des genres littéraires dont elles relèvent, et les usages linguistiques dont elles dépendent doivent être analysés et déconstruits. Surtout, cette critique interne doit s'appliquer tant aux textes originels qu'aux discours produits à partir de ces derniers par les philologues qui les ont découverts, traduits et commentés. C'est ce qu'Oliver Wolters s'est proposé de faire dans les quatre articles fondamentaux cités plus haut (et qu'il a continué de faire dans le domaine des études vietnamiennes).

Si les historiens et les archéologues éprouvent depuis les années quatre-vingt le besoin de déconstruire ainsi les travaux sur l'Asie du Sud-Est des grands ancêtres orientalistes, c'est bien que leur œuvre, à tant d'égards remarquable, souffre néanmoins d'une contra-

5. Il faut préciser néanmoins qu'Oliver Wolters a vécu avant-guerre de nombreuses années dans la Malaisie voisine. Ce n'est donc pas, dans ces années quatre-vingt, une découverte naïve des paysages exotiques qui s'opère sur ce nouveau terrain.

6. Wolters, 1986.

7. Westenenk, 1921, 1923. 
diction originelle: ainsi, pour ne prendre que l'exemple de cette EFEO centenaire qui nous occupe ici, on se doit de constater qu'elle a bien franchi en pionnière le fossé qui séparait le texte du terrain, et qu'elle s'est donné les moyens d'étudier l'Asie du Sud-Est sur place ; mais elle a fourni ces moyens à des savants qui n'ont pas avant les années cinquante ou soixante pris toute la mesure des faits de civilisation propres à la région qu'ils étudiaient, quelle que soit leur discipline d'origine. Sinologues ou indianistes, formés pour la plupart à un orientalisme pour lequel les civilisations des deux grands voisins de l'Asie du Sud-Est sont prééminentes, mais aussi savants d'une époque coloniale, insérés dans les grands paradigmes du temps dans lequel ils inscrivent leur réflexion, ils lisent les textes qu'ils découvrent (sources chinoises et épigraphie sanskrite pour l'essentiel) en les passant aux cribles accumulés de plusieurs civilisations étrangères à la région. De fait, le parti pris des savants orientalistes de la première moitié du $\mathrm{XX}^{\mathrm{c}}$ siècle est tout entier en germe dans la phrase lapidaire prononcée par Louis Finot en 1901, devant l'Académie des inscriptions et belles-lettres: "L'Indochine ne s'explique pas par elle-même ${ }^{8}$.

Pour pouvoir déconstruire le discours de ces pionniers des études sud-est asiatiques, il a donc fallu admettre, d'abord, que l'Asie orientale, prise globalement, pouvait avoir vécu des expériences autres que celles de la Mésopotamie ou de l'Europe, dans son cheminement vers des formes qualitativement distinctes et multiples d'interaction et d'intégration sociales, comme dans ses rapports à la nature, à l'espace ; puis, dans un deuxième temps, il a fallu admettre aussi que l'Asie du Sud-Est, au sein de l'Asie orientale, devait être considérée pour elle-même, et non pas comme une "Indo-Chine » ou une "Insulinde » qui ne serait qu'une somme d'influences reçues de ses prestigieux voisins.

Ce n'est pas ici le lieu d'aborder, dans son ensemble, ce vaste débat. Je voudrais seulement, avec l'exemple des études sur Sriwijaya, pris dans le domaine de l'archéologie historique, en fournir quelques illustrations qui me semblent éloquentes.

Si l'on avait déjà repéré dès avant la fin du XIX $X^{e}$ siècle, dans les sources chinoises et arabes, un nom d'État que Pelliot reconstituait encore en 1904 sous la forme Śríbhoja, on sait que c'est à George Cœedès que l'on doit "l'invention » de Sriwijaya. Dans le génial article qu'il donna au Bulletin de l'École française d'Extrême-Orient de 1918, il mettait en rapport les sources chinoises et arabes, l'épigraphie indienne et, pour la première fois, une inscription en vieux malais datée de 686, trouvée à Sumatra-Sud : en y reconnaissant toujours des références à un même système politique, il signait en quelque sorte l'acte de naissance de ce qu'il nomma le «royaume de Śrīijaya ». Il continuera longtemps de publier des études sur la question, mais jamais, soit dit en passant, il ne se rendra sur le terrain sumatranais.

La question des origines et de la structure de ce grand système politique a fait depuis l'objet de débats passionnés, souvent contradictoires. Celle de la localisation de sa première capitale, en particulier, a fait couler beaucoup d'encre. George Cœdès, comme Paul Pelliot avant lui, proposait de la situer à Palembang, grande ville portuaire de Sumatra-Sud, de nos jours encore ; ce faisant, il défendait une hypothèse de bon sens, qui s'accordait au mieux avec les sources chinoises et l'épigraphie malaise du vIl ${ }^{\mathfrak{c}}$ siècle. Il fut suivi dans cette hypothèse par la meilleure part des historiens « vétérans »; d'autres, pas toujours exempts de sentiments nationalistes, proposèrent néanmoins pour abriter la capitale de cet État prestigieux des sites multiples, ailleurs à Sumatra, mais aussi en

8. Finot, 1901, p. 6 du tiré à part.

9. Pelliot, 1904, p. 336-337; Cœdès, 1918, $1930,1964$. 
Malaisie ou en Thaïlande méridionale, toutes régions dont il est clair qu'elles avaient, à un titre ou à un autre, été intégrées dans la vaste sphère d'influence de Sriwijaya ${ }^{10}$.

Progressivement, au fur et à mesure que les sources textuelles qui en faisaient mention s'accumulaient, l'importance, le poids historique de cet État présumé malais allait en croissant. On se trouva ainsi bientôt confronté à un système politique qui avait duré environ six siècles (fait exceptionnel dans la région), à un État dont les richissimes souverains s'attiraient le respect de leurs pairs - depuis la Bagdad des Mille et une nuits jusqu'aux non moins riches capitales de l'Empire du Milieu -; ces mêmes souverains faisaient dresser en leur nom des inscriptions aussi bien à Canton qu'à Nalanda ou en pays Cola, et entretenaient dans leur capitale une vaste communauté de moines bouddhistes dont le rayonnement devait marquer les développements de cette religion aussi bien en Chine qu'au Tibet.

La controverse à propos de Sriwijaya, pourtant, se prolongeait. L'embarras des promoteurs de sa localisation à Palembang tenait à un «détail » de poids : la cité capitale de ces souverains restait introuvable sur le terrain archéologique (à Sumatra comme d'ailleurs sur les autres sites proposés, qui ne tiraient pas mieux leur épingle de ce jeu). Pas la moindre petite trace archéologique à Sumatra-Sud d'une agglomération comparable, par exemple, à la cité angkorienne ; pas même, faute de vestiges civils, de concentrations de monuments religieux construits en dur, dont on se contentait alors en général, de Pagan à Java Central, pour localiser un "royaume ». Il n'est pas inutile de rappeler ici, par parenthèse, que les archéologues cherchent encore la (ou les) ville(s) dont les souverains bâtirent à Java les remarquables monuments du Borobudur ou de la plaine de Prambanan, contemporains de Sriwijaya.

À Sumatra-Sud, en dehors des inscriptions en vieux malais du $\mathrm{VII}^{\mathrm{e}}$ siècle, dont la simple localisation comme le contenu continuaient d'affirmer avec entêtement qu'on était bien là au cœur géographique et politique du pouvoir, on arrivait au mieux à regrouper quelques briques éparses et deux douzaines de statues dépareillées, fascinantes certes, mais difficiles à classer pour les historiens de l'art habitués à détecter des styles à l'évolution plus cohérente. Une équipe d'archéologues professionnels, après deux mois de prospections et de fouilles à Palembang en 1974, en arriva même à décréter que rien n'y signalait la présence avant le $\mathrm{XIV}^{\mathfrak{c}}$ siècle d'une concentration urbaine plus grosse qu'un simple village. On en était là quand Satyawati Sulaiman et Oliver Wolters décidèrent de relancer les recherches sur les bases nouvelles auxquelles j'ai déjà fait allusion.

En simplifiant à l'extrême, les présupposés fondés sur les premières lectures des sources textuelles étaient de trois ordres :

- Un souverain qui, du fait de son contrôle quasi exclusif de la grande route maritime transasiatique et de ses trafics, était un interlocuteur économique incontournable en Asie, ne pouvait qu'avoir gouverné un "empire», dont le «territoire» - placé sous son contrôle direct ou sous celui de souverains "vassaux »-, les "provinces » et les autres divisions administratives se devaient d'être clairement circonscrites dans l'espace sud-est asiatique.

- Sa riche capitale ne pouvait qu'être «fortifiée » ou "murée ", comme le laissait entendre à tort la première traduction anglaise du texte du pèlerin Yijing par le bouddhisant japonais Takakusu Junjirô, pas mieux à l'abri dans son Japon natal (et, pour nous, extrême-oriental) des interprétations biaisées des orientalistes occidentaux ${ }^{11}$. 
- Enfin, une communauté de moines bouddhistes aussi prestigieuse ne pouvait qu'avoir laissé derrière elle les vestiges de monuments religieux nombreux, construits en dur. Pour expliquer cette dernière carence, à ses yeux incompréhensible, George Codès en vint même à mettre en doute les vertus spirituelles des souverains de Sriwijaya, qui, préoccupés de basses questions économiques, n'auraient probablement pas eu le temps ou le goût d'élever des monuments à la gloire divine.

Il fallait donc s'extraire de ces discours narratifs exclusifs, qui avaient été entretenus pendant des décennies par les historiens, les philologues et, à leur suite, par les archéologues. En résumé, et en grossissant le trait, il fallait cesser d'être préoccupés, comme nos prédécesseurs, par la quête du bâti monumental, censé inscrire dans l'espace les signes de la supériorité hiérarchique d'un pouvoir politique et économique fort. Il fallait rompre avec cette recherche quasi obsessionnelle des signes de la permanence sur le terrain sumatranais, préoccupation qui en arrivait à rendre invisible à l'archéologue nombre de concentrations urbaines (à Palembang comme sur bien d'autres sites de l'Asie du Sud-Est, soit dit en passant) ${ }^{12}$.

Sous la poussée amicale d'Oliver Wolters et de Satyawati Suleiman, nous avons donc été quelques-uns, historiens et archéologues indonésiens ou occidentaux, spécialistes déjà de la région, à prendre le relais que l'on nous passait ainsi $i^{13}$.

À partir du moment où nous posions comme hypothèse de départ que les vestiges de cet État et de sa cité portuaire, siège de son pouvoir politique, étaient situés dans le sud de l'île de Sumatra, au cœur du monde malais, il nous fallait tenter de construire, en suivant certaines des pistes ouvertes par les travaux d'Oliver Wolters, un cadre de référence mieux adapté au milieu humain et à l'environnement dans lequel nous pensions que l'État de Sriwijaya était né au VII ${ }^{\mathrm{c}}$ siècle.

En d'autres termes, il nous fallait achever de parcourir le long trajet qui devait nous mener à la compréhension d'un terrain véritablement malais. Nous y avons largement été aidés par les recherches menées, parallèlement à celles des archéologues, par de nombreux historiens modernistes (nombre d'entre eux élèves ou proches aussi d'Oliver Wolters) ${ }^{14}$. Nous en sommes arrivés progressivement à la conclusion que les fameuses villes marchandes du monde malais, infiniment mieux documentées pendant le moment islamique de leur histoire, entre les $\mathrm{XV}^{\mathrm{c}}$ et $\mathrm{XVII}^{\mathrm{e}}$ siècles, pouvaient nous fournir des modèles de référence utiles à la compréhension de la formation de l'État et de l'urbanisation pour les périodes précédentes.

Or ces cités-États ne constituaient pas des systèmes politiques stables, centralisés, comme on en connaît sur le pourtour méditerranéen, en Chine et en Inde, avec des villes construites en dur, gouvernant des territoires. S'il s'agissait d'États indéniablement efficaces économiquement, au centre d'espaces sociaux très étendus, ils étaient intrinsèquement instables dans l'espace, s'organisant politiquement autour de centres portuaires alternatifs.

De ce fait, ces États n'ont jamais construit de villes, si grandes et riches soient-elles, qui laissent des vestiges archéologiques facilement décelables sur le terrain. Ceci alors même qu'elles pouvaient constituer un passage obligé pour l'ensemble des marchands et des religieux qui parcouraient la grande route maritime qui relie la Chine à la

12. Voir aussi McIntosh, 1999, p. 65-66, 72-73, pour une problématique comparable appliquée à la formation de l'État et à l'urbanisation de la Chine archaïque et de l'Afrique médiévale.

13. Voir, en bibliographie, les travaux de Lucas, Soeroso \& Manguin, McKinnon, Manguin, Suleiman.

14. On se reportera, entre autres, aux travaux des auteurs suivants, dont les titres sont donnés dans la bibliographie : Christie, Kathirithamby-Wells \& Villiers, Kulke, Lombard, Manguin, Miksic, Milner, Reid, Sandhu \& Wheatley. 
Méditerranée. Ainsi, sans les textes malais, chinois et portugais contemporains, la fameuse Melaka malaise des $\mathrm{XV}^{\mathrm{e}}$ et $\mathrm{XVI}^{\mathrm{e}}$ siècles, malgré sa population de quelque 100000 habitants, n'aurait jamais pu être décrite par les seuls archéologues d'antan.

Les anciennes cités-Etats portuaires du monde malais ont été construites en bois, dressées sur pilotis, pour l'essentiel sur les rives mouvantes des rivières. Elles n'étaient pas entourées d'enceintes permanentes, moins encore « murées », mais s'inscrivaient sans le modifier durablement dans leur environnement ripuaire et se fondaient à leur périphérie dans un paysage "rurbain ". Les Malais, contrairement à leurs voisins khmers, n'ont pas tenté de modeler, de géométriser l'espace dans lequel ils vivaient. Au mieux, ils aménageaient un site naturel existant.

Les magnifiques palais de leurs richissimes souverains étaient entourés d'une simple enceinte en bois ; ils étaient de même construits en bois et décorés d'or et de tissus, toutes matières réutilisables ou périssables. Seuls quelques monuments religieux étaient construits en dur, sur des éminences à l'abri des marées et des crues : mais, faute de pierre dans l'environnement où se dressaient habituellement ces villes, les Malais ont toujours fait appel aux briques et au décor en terra cotta, dont la ruine a été rapide et les matériaux constamment réutilisés, jusqu'à nos jours.

Le temps me manque ici malheureusement pour vous fournir la preuve tangible de ce que je vous affirme ainsi à propos de Sriwijaya, pour vous démontrer, photos à l'appui, que les campagnes archéologiques menées depuis 1989 par les archéologues indonésiens, en coopération avec ceux de l'École française d'Extrême-Orient, ont bien porté leurs fruits. Car, sans avoir, loin s'en faut, résolu tous les problèmes qui se posaient, nous avons néanmoins pu apporter la preuve archéologique que la cité-État de Sriwijaya avait bien été fondée sur le site de la ville de Palembang, qu'elle y avait prospéré jusqu'au $\mathrm{XI}^{\mathrm{e}}$ siècle, et survécu bien au-delà. À force de patience, nous y avons retrouvé les vestiges, ténus et fragiles mais incontournables, d'un habitat ripuaire proche de celui que l'on peut encore observer de nos jours à Sumatra, ou encore à Bornéo. Nous avons mis au jour par dizaines de milliers, dans la vase des anciennes rives des rivières et des arroyos, les tessons de céramique témoignant de la vie quotidienne des habitants et de leurs échanges hauturiers : poteries fabriquées localement, mais aussi venues du golfe Persique, de l'Inde et, surtout, de la Chine méridionale. Nous avons retrouvé enfin les vestiges d'une vingtaine de sites de l'arrière-pays du bassin fluvial passé sous le contrôle de la place centrale de Palembang, avec leurs temples en brique, qui marquaient les principaux confluents du réseau hydrographique du fleuve Musi.

En somme, comme nous avait suggéré de le faire Oliver Wolters, nous avons appris à déchiffrer le discours qui nous était proposé par l'environnement naturel des basses terres de Sumatra et par l'espace que s'y étaient aménagé les Malais de Sriwijaya. Ces signes, que nous avons appris à lire sur le terrain, nous avons alors pu constater qu'ils étaient déjà suggérés par les textes. Mais il fallait d'abord avoir réappris à les lire.

\section{Références bibliographiques}

CHRISTIE, J. Wisseman

1990 «Trade and state formation in the Malay Peninsula and Sumatra, 300 B.C.A.D. 700 », in J. Kathirithamby-Wells \& J. Villiers, éd., The Southeast Asian Port and Polity: Rise and Demise, Singapore, Singapore University Press, p. 39-60.

1995 "State formation in Early Maritime Southeast Asia: A consideration of the theories and the data $", B K I, 151$, p. $235-288$. 
CEDÈs, G.

1918 «Le Royaume de Çrîvijaya », BEFEO, 18, p. 1-36.

1930 «Les inscriptions malaises de Çrîvijaya », BEFEO, 30, p 29-80.

1964 Les États hindouisés d'Indochine et d'Indonésie, Paris, Éditions de Boccard [3 $3^{\mathrm{e}}$ éd.].

FINOT, L.

1901 «Compte rendu sur l'état de l'École française d'Extrême-Orient», Académie des inscriptions et belles-lettres, Comptes rendus des séances de l'année 1901, tiré à part.

KATHIRITHAMBY-WELlS, J. \& Villiers, J. (éd.)

1990 The Southeast Asian Port And Polity: Rise And Demise, Singapore, Singapore University Press.

KULKE, $\mathrm{H}$.

1995 « Kadatuan Srivijaya - Empire or Kraton of Srivijaya? A Reassessment of the epigraphical evidence ", BEFEO, 80, p. 159-181.

LuCas, P. K., Manguin, P.-Y. \& Soeroso

1998 «Kota Kapur (Bangka, Indonesia): A pre-Sriwijayan site reascertained », in P.-Y. Manguin, éd., Southeast Asian Archaeology 1994, Hull, University of Hull, Centre for Southeast Asian Studies, vol. II, p. 61-81.

LOMBARD, D.

1988 «Le sultanat malais comme modèle socio-économique ", in D. Lombard \& J. Aubin, éd., Marchands et hommes d'affaires asiatiques dans l'Océan Indien et la Mer de Chine, 13e-20e siècles, Paris, Éditions de l'EHESS, p. 117-125.

1990 Le carrefour javanais : essai d'histoire globale, Paris, Éditions de l'EHESS (Civilisations et sociétés, 79), 3 vol.

1994 « $\dot{A}$ propos de l'histoire des villes d'Asie du Sud-Est. Nouvelles considérations », in P. Clément, S. Clément-Charpentier \& C. Goldblum, éd., Cités d'Asie, Paris, Éditions Parenthèses (Les cahiers de la recherche architecturale, 35-36), p. 99-106.

MANGUIN, P.-Y.

1987 «Palembang et Sriwijaya: anciennes hypothèses, nouvelles recherches (Palembang Ouest) », BEFEO, 76, p. 337-402.

1991 "The Merchant and the King: Political myths of Southeast Asian coastal polities », Indonesia, 52, p. 41-54.

1992 «Excavations in South Sumatra, 1988-1990: New evidence for Sriwijayan sites », in I. C. Glover, éd., Southeast Asian Archaeology 1990, Hull, University of Hull, Centre for Southeast Asian Studies, p. 63-73.

1993a «Sriwijaya and the early trade in Chinese ceramics: observations on recent finds from Palembang (Sumatra) ", in Report, UNESCO Maritime Route of Silk Roads, Nara Symposium '91, Nara, The Nara International Foundation, p. 122-133.

1993b "Palembang and Sriwijaya: An Early Malay Harbour-city rediscovered", Journal of the Malaysian Branch, Royal Asiatic Society, 66(1), p. 23-46.

1999 «Demografi dan tata perkotaan di Aceh pada abad 16: Data baru menurut sebuah buku pedoman Portugis tahun $1584 »$ [Démographie et urbanisme à Aceh au $\mathrm{XVI}^{\mathrm{C}}$ siècle : nouvelles données d'après un Roteiro portugais de 1584], in H. Chambert-Loir \& Hasan Muarif Ambary, éd., Panggung 
Sejarah: Persembahan kepada Prof. Dr. Denys Lombard, Jakarta, EFEO/ Pusat Penelitian Arkeologi Nasional/Yayasan Obor Indonesia, p. 225-244.

2000 «Les cités-États de l'Asie du Sud-Est côtière: de l'ancienneté et de la permanence des formes urbaines », BEFEO, 87(1), p. 151-182.

sous pressela "The amorphous nature of coastal polities in Insular Southeast Asia: Restricted centres, extended peripheries ", in M. Charras, éd., Beyond the State: Socio-spatial structuration in Southeast Asia, Paris, LASEMA-CNRS.

sous presse/b "Srivijaya dans l'œuvre de George Cœdès », in L. Gabaude, éd., George Cœdès aujourd'hui, Bangkok, Centre d'Anthropologie Sirindhorn (Centre de documentation et de recherches d'Études franco-thaïes).

MCINTOSH, R. J.

1999 "Clustered cities and alternative courses to authority in prehistory", Journal of East Asian Archeology, 1(1-4), p. 63-85.

MCKINNON, E. E.

1992 «Malayu Jambi: interlocal and international trade (11th to 13th centuries)», in Seminar Sejarah Malayu Kuno, Jambi 1992, Jambi, Pemerintah Daerah Tingkat I Jambi / Kantor Wilayah Departemen Pendidikan dan Kebudayaan Jambi, p. 336-375.

MCKINNON, E. E. \& MiLNER, A.

1979 «A letter from Sumatra: A visit to some early Sumatran historical sites», Indonesia Circle, 18, p. 2-21.

MIKSIC, J. N.

1989 «Urbanization and social change: the case of Sumatra », Archipel, 37, p. 3-29.

1993-94 «Recent research at Trowulan: Implications for Early Urnanization in Indonesia ", in Pertemuan Ilmiah Arkeologi VI, Batu, Malang, 26-29 Juli 1992, Jakarta, Pusat Penelitian Arkeologi Nasional, p. 357-366.

1995 «Evolving archaeological perspectives on Southeast Asia, 1970-75 », Journal of Southeast Asian Studies, 26, p. 46-62.

MILNER, A.

1982 Kerajaan: Malay political culture on the eve of colonial rule, Tucson, University of Arizona Press.

PELLIOT, P.

1904 «Deux itinéraires de Chine en Inde à la fin du VIII ${ }^{\mathrm{e}}$ siècle », $B E F E O, 4$, p. 131-413.

REID, A.

1980 «The structure of cities in Southeast Asia, Fifteenth to Seventeenth Centuries ", Journal of Southeast Asian Studies, 11(2), p. 235-250.

1988-93 Southeast Asia in the Age of Commerce, 1450-1680, Volume one: The Lands below the Winds, Volume two: Expansion and crisis, New Haven / London, Yale University Press.

1999 Charting the shape of Early Modern Southeast Asia, Chiang Mai, Silkworm Books.

2000 «Negeri. The Culture of Malay-Speaking City-states of the Fifteenth and Sixteenth Centuries ", in M. H. Hansen, éd., A comparative study of thirty City-State Cultures: An investigation conducted by the Copenhagen Polis Centre, Copenhagen, Historisk-filosofiske Skrifter, The Royal Danish Academy of Sciences and Letters, p. 417-430. 
REID, A. (éd.)

1993 Southeast Asia in the Early Modern Era: Trade, Power, and Belief, Ithaca, Cornell University Press.

SANDhU, K. S. \& Wheatley, P.

1983 Melaka: The transformation of a Malay capital c. 1400-1980, Oxford / Kuala Lumpur, Oxford University Press, 2 vol.

SULEIMAN, Satyawati

1980 "The History and Art of Srivijaya », in Subhadradis Diskul, éd., The Art of Srivijaya, Paris / Kuala Lumpur, UNESCO / Oxford University Press, p. 1-20.

1981 "Research on Srivijaya history and art history », in Studies on Sriwijaya, Jakarta, National Research Centre of Archaeology, p. 53-70.

TAKAKUSU JUNJIRÔ

1896 A Record of Buddhist Religion as Practised in India and the Malay Archipelago (A.D. 671-695) by I-Tsing, Oxford, Clarendon Press.

TAYLOR, K. W.

1992 "The early kingdoms », in N. Tarling, éd., The Cambridge History of Southeast Asia. Volume one: From Early Times to c. 1800, Cambridge, Cambridge University Press, p. 137-182.

WESTENENK, L. C.

1921 «Uit het Land van Bittertong (Zuid-Soematra)», Djåwå, 1(1), p. 5-11.

1923 «Boekit Segoentang en Goenoeng Mahaméroe uit de Sedjarah Melajoe », $T B G, 68(1)$, p. $212-226$.

WOLTERS, O. W.

1967 Early Indonesian Commerce: A Study of the Origins of Sri Vijaya, Ithaca, Cornell University Press.

1970 The Fall of Srivijaya in Malay History, Kuala Lumpur / Singapore, Oxford University Press.

1975 «Landfall on the Palembang Coast in Medieval Times », Indonesia, 20, p. 1-57.

1979a "Studying Srivijaya", Journal of the Malayan Branch, Royal Asiatic Society, 52(2), p. 1-32.

$1979 \mathrm{~b}$ «A Note on Sungsang Village at the Estuary of the Musi River in Southeastern Sumatra: A Reconsideration of the Historical Geography of the Palembang Region », Indonesia, 27, p. 33-50.

1984 «Palembang Revisited», Southeast Asia Program Bulletin (Cornell University), Year 1984, p. 17-18.

1986 «Restudying Some Chinese Writings on Sriwijaya », Indonesia, 42, p. 1-42. 